\title{
Statistical covariance-matching based blind channel estimation for zero-padding MIMO-OFDM systems
}

Yi-Sheng Chen ${ }^{1 *}$ and Jwo-Yuh Wu ${ }^{2}$

\begin{abstract}
We propose a statistical covariance-matching based blind channel estimation scheme for zero-padding (ZP) multipleinput multiple-output (MIMO)-orthogonal frequency division multiplexing (OFDM) systems. By exploiting the block Toeplitz channel matrix structure, it is shown that the linear equations relating the entries of the received covariance matrix and the outer product of the MIMO channel matrix taps can be rearranged into a set of decoupled groups. The decoupled nature reduces computations, and more importantly guarantees unique recovery of the channel matrix outer product under a quite mild condition. Then the channel impulse response matrix is identified, up to a Hermitian matrix ambiguity, through an eigen-decomposition of the outer product matrix. Simulation results are used to evidence the advantages of the proposed method over a recently reported subspace algorithm applicable to the ZP-based MIMO-OFDM scheme.
\end{abstract}

Keywords: Blind channel estimation, Zero padding, MIMO-OFDM

\section{Introduction}

Orthogonal frequency division multiplexing (OFDM) combined with guard intervals, in the form of cyclic prefix (CP) or zero-padding (ZP), is an effective transmission scheme through frequency selective fading channels [1]. By further leveraging the spatial resource, the multipleinput multiple-output (MIMO)-OFDM system has been the key technique for realizing high-rate transmission in modern wireless communications [2]. Toward reliable coherent symbol decoding in MIMO-OFDM systems, accurate channel state information is crucial. Blind channel estimation is a technique that alleviates the need for training sequences to identify the unknown channel impulse response from the received signal. Since the requirement of extra bandwidth for training overhead is reduced, this technique has received great research interest [3] and many blind estimation algorithms have been developed for various transmission systems [3-21]. In this article, we will focus on blind estimation of ZP-based MIMO-OFDM systems.

*Correspondence: yischen@fcu.edu.tw

${ }^{1}$ Feng Chia University, Taichung, Taiwan

Full list of author information is available at the end of the article
For ZP-based single-input single-output (SISO) OFDM systems, a subspace algorithm is proposed to blindly identify the channels in [20], and is then generalized to MIMO cases [21]. However, this approach is known to suffer a sever performance degradation when the signal-to-noise ratio (SNR) is low or moderate [5]. To solve this problem, a statistical covariance-matching (SCM) based method which exploits some priori knowledge of the signal structure to improve channel estimation/equalization performances against harsh SNR conditions, is developed for SISO cases [6]. In this article we will propose an SCM based blind channel estimation for ZP-based MIMOOFDM systems. By exploiting the block Toeplitz channel matrix structure, we show that the linear equations relating the entries of the received covariance matrix and the products of the channel matrix taps can be rearranged into decoupled groups. The outer product of the MIMO channel matrices can be obtained by solving these decoupled linear equation groups. The channel impulse response is then identified, up to a Hermitian matrix ambiguity, through an eigen-decompostion of the computed outer product matrix. The proposed approach has the following distinctive features: (i) The identifiability condition is very simple and is more relaxed than the 
irreducible or column reduced condition [8]; (ii) It can apply to the more transmit antennae case under a certain condition; (iii) Through numerical simulation, it yields improved BER performance in the low-to-moderate SNR region. The rest of this article is organized as follows. Section 2 is the system model and basic assumptions. In Section 3, we propose a blind channel estimation method for the ZP based MIMO-OFDM systems. Simulation results are given in Section 4. Section 5 concludes this article.

Notations used in this article are quite standard: Bold uppercase is used for matrices, and bold lowercase is used for vectors. $\mathbf{A}^{T}$ represents transpose of the matrix $\mathbf{A}$, and $\mathbf{A}^{*}$ represents conjugate transpose of the matrix A. $\mathbf{I}_{M}$ is the identity matrix of dimension $M \times M$, and $\mathbf{A} \otimes \mathbf{B}$ is the Kronecker product of matrices $\mathbf{A}$ and $\mathbf{B}$. In addition, we define the following operations that will be used in the derivation of the main result. First, for any $m \times m$ matrix $\mathbf{A}=\left[a_{k, l}\right]_{0 \leq k, l \leq m-1}$, define $\Gamma_{j}(\mathbf{A})=$ $\left[a_{0, j} a_{1, j+1} \ldots a_{m-1-j, m-1}\right]^{T}$ for $0 \leq j \leq m-1$, i.e., $\Gamma_{j}(\mathbf{A})$ is the vector formed from the $j$ th super-diagonal of $\mathbf{A}$. Second, for any $J n \times J n$ matrix $\mathbf{B}=\left[\mathbf{B}_{k, l}\right]_{0 \leq k, l \leq n-1}$, where $\mathbf{B}_{k, l}$ is a block matrix of dimension $J \times J$, define $\Upsilon_{j}(\mathbf{B})=$ $\left[\mathbf{B}_{0, j}^{T} \mathbf{B}_{1, j+1}^{T} \ldots \mathbf{B}_{n-1-j, n-1}^{T}\right]^{T}$ for $0 \leq j \leq n-1$, i.e., $\Upsilon_{j}(\mathbf{B})$ is the matrix formed from the $j$ th block super-diagonal of $\mathbf{B}$.

\section{System model and basic assumptions}

Consider the $K$-input $J$-output discrete time ZP-OFDM baseband model shown in Figure 1. At the transmitter, for $k=1,2, \ldots, K$, each input signal $s_{k}(n)$ is stacked as a block $\overline{\mathbf{s}}_{k}(i)=$ $\left[s_{k}(i M) s_{k}(i M+1) \ldots s_{k}(i M+M-1)\right]^{T} \in \mathbb{C}^{M}$, which is multiplied by the inverse FFT matrix $\mathbf{F}^{*}$, and then padded with $P$ trailing zero entries to form the $N=M+P$ dimensional vector $\overline{\mathbf{u}}_{k}(i)=[\underbrace{\left(\mathbf{F}^{*} \overline{\mathbf{s}}_{k}(i)\right)^{T}}_{M \text { entries }} \underbrace{0 \ldots 0}_{\text {Pentries }}]^{T}=$ $[\underbrace{u_{k}(i N) \ldots u_{k}(i N+M-1)}_{M \text { entries }} \underbrace{0 \ldots 0}_{P \text { entries }}]^{T}$. The zero-padded $\overline{\mathbf{u}}_{k}(i)$ is parallel-to-serial converted to obtain $u_{k}(n)$, which is then transmitted through the MIMO finite-impulseresponse (FIR) channel. At the receiver, the $j$ th received signal is $x_{j}(n)=\sum_{k=1}^{K} \sum_{l=0}^{L_{j k}} h_{j k}(l) u_{k}(n-l)+w_{j}(n)$ for $j=1,2, \ldots, J$, where $w_{j}(n)$ is the channel noise seen at the $j$ th receiver, and $\left\{h_{j k}(0) h_{j k}(1) \ldots h_{j k}\left(L_{j k}\right)\right\}$ is the impulse response from the $k$ th transmitter to the $j$ th receiver.

Let $\mathbf{x}(n)=\left[x_{1}(n) x_{2}(n) \ldots x_{J}(n)\right]^{T} \in \mathbb{C}^{J}, \mathbf{w}(n)=$ $\left[w_{1}(n) w_{2}(n) \ldots w_{J}(n)\right]^{T} \in \mathbb{C}^{J}$, and $\mathbf{H}(l)=\left[h_{j k}(l)\right] \in \mathbb{C}^{J \times K}$ be the channel coefficient matrix for $l=0,1, \ldots, L$, where $L=\max _{j, k}\left\{L_{j k}\right\}$ is the order of the MIMO channel. Assume $P \geq L$ and group the sequence of $\mathbf{x}(n)$ as $\overline{\mathbf{x}}(i)=\left[\mathbf{x}(i N)^{T} \mathbf{x}(i N+1)^{T} \ldots \mathbf{x}(i N+N-1)^{T}\right]^{T} \in \mathbb{C}^{J N}$. Then due to zero padding, the input-output channel characteristics can be expressed in the following form [21]:

$$
\overline{\mathbf{x}}(i)=\mathbf{H}_{f} \mathbf{u}_{f}(i)+\overline{\mathbf{w}}(i),
$$

where $\overline{\mathbf{w}}(i) \in \mathbb{C}^{N}$ is similarly defined as $\overline{\mathbf{x}}(i)$, and $\mathbf{H}_{f} \in \mathbb{C}^{N \times K M}$ is a block Toeplitz matrix with $\left[\mathbf{H}(0)^{T} \mathbf{H}(1)^{T} \ldots \mathbf{H}(L)^{T} \mathbf{0} \ldots \mathbf{0}\right]^{T} \in \mathbf{C}^{J \times \times K}$ being its first block column and $[\mathbf{H}(0) \mathbf{0} \ldots \mathbf{0}] \in \mathbf{C}^{J \times K M}$ being its first block row. $\mathbf{u}_{f}(i)=\left[\mathbf{u}(i N)^{T} \mathbf{u}(i N+1)^{T} \ldots \mathbf{u}(i N+M-\right.$ $\left.{ }^{1)^{T}}\right]^{T} \in \mathbb{C}^{K M}$ with $\mathbf{u}(n) \in \mathbb{C}^{K}$ being similarly defined as $\mathbf{x}(n)$.

The problem we study in this article is blind estimation of the MIMO channel matrix taps $\mathbf{H}(m), 0 \leq m \leq L$, by using second-order statistics of the received data. The following assumptions hold throughout the article.

(A) The source signal $\mathbf{s}(n)=\left[s_{1}(n) s_{2}(n) \ldots s_{K}(n)\right]^{T} \in \mathbb{C}^{K}$ is a zero mean white sequence with $E\left[\mathbf{s}(m) \mathbf{s}(n)^{*}\right]=$ $\delta(m-n) \mathbf{I}_{K}$, where $\delta(\cdot)$ is the Kronecker delta function. The noise is white zero mean with $E\left[\mathbf{w}(m) \mathbf{w}(n)^{*}\right]=$ $\delta(m-n) \sigma_{w}^{2} \mathbf{I}_{j}$. In addition, the source signal is

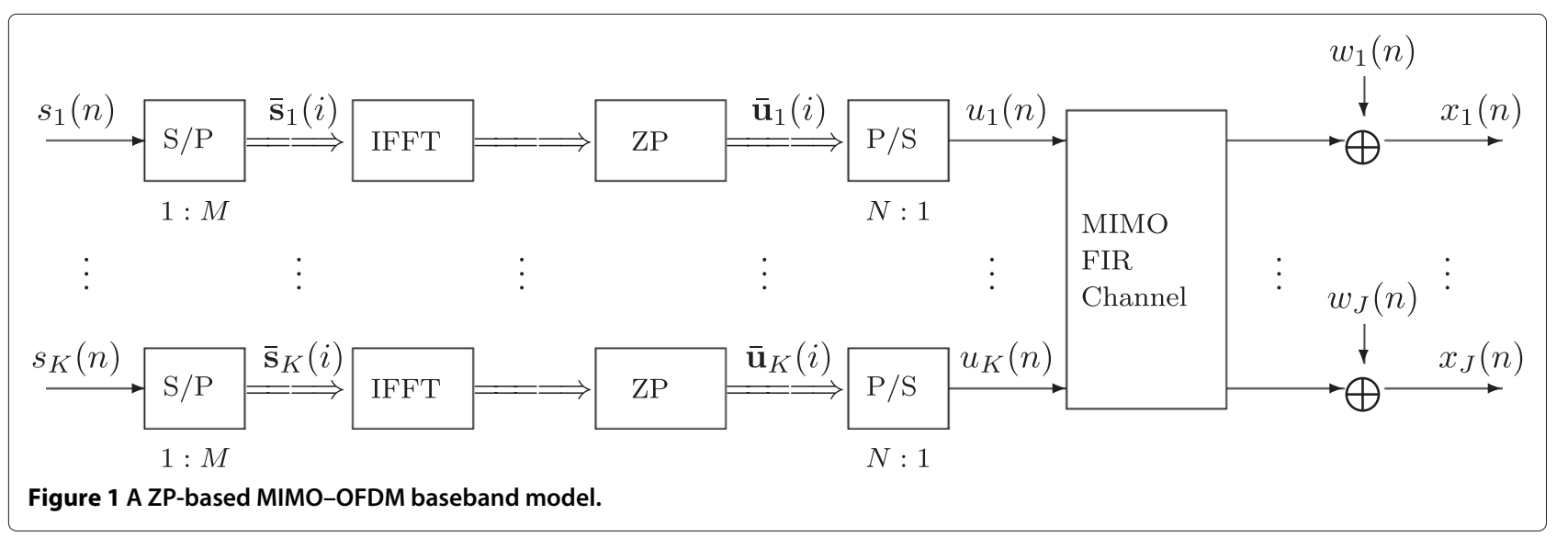


uncorrelated with the noise $\mathbf{w}(n)$, i.e., $E\left[\mathbf{s}(m) \mathbf{w}(n)^{*}\right]=$ $\mathbf{0}_{K \times J}, \forall m, n$.

(B) The concatenated channel impulse response matrix $\mathbf{H}=\left[\mathbf{H}(0)^{T} \mathbf{H}(1)^{T} \ldots \mathbf{H}(L)^{T}\right]^{T} \in \mathbb{C}^{J(L+1) \times K}$ is full column rank, i.e., $\operatorname{rank}(\mathbf{H})=K$.

\section{Blind channel estimation}

We first introduce the proposed method, assuming the noise is absent in Section 3.1; the case when noise is present and some distinctive features regarding the proposed method are discussed in Section 3.2.

\subsection{Proposed approach: noiseless case}

When noise is absent, (2.1) can be expressed as $\overline{\mathbf{x}}(i)=$ $\mathbf{H}_{f} \mathbf{u}_{f}(i)$. By further defining the block source signal $\mathbf{s}_{f}(i)=\left[\mathbf{s}(i M)^{T} \mathbf{s}(i M+1)^{T} \ldots \mathbf{s}(i M+M-1)^{T}\right]^{T} \in \mathbb{C}^{K M}$, we have $\mathbf{u}_{f}(i)=\left(\mathbf{F}^{*} \otimes \mathbf{I}_{K}\right) \mathbf{s}_{f}(i)$ [9], which is a zero mean vector with $E\left[\mathbf{u}_{f}(i) \mathbf{u}_{f}(i)^{*}\right]=\left(\mathbf{F}^{*} \otimes \mathbf{I}_{K}\right)\left(\mathbf{F}^{*} \otimes \mathbf{I}_{K}\right)^{*}=\left(\mathbf{F}^{*} \mathbf{F}\right) \otimes$ $\left(\mathbf{I}_{K} \mathbf{I}_{K}\right)=\mathbf{I}_{K M}$ according to assumption (A). Then taking expectation of $\overline{\mathbf{x}}(i) \overline{\mathbf{x}}(i)^{*}$, we get

$$
\mathbf{R}_{f}=E\left[\overline{\mathbf{x}}(i) \overline{\mathbf{x}}(i)^{*}\right]=\mathbf{H}_{f} \mathbf{H}_{f}^{*}
$$

Let $\mathbf{J} \in \mathbb{R}^{N \times N}$ be a circulant matrix with the first row equal to $[00 \ldots 01] \in \mathbb{R}^{1 \times N}$ and $\mathbf{S}=\left[\mathbf{I}_{N-L} \mathbf{0}_{(N-L) \times L}\right]^{T} \in$ $\mathbb{R}^{N \times(N-L)}$. Then the block Toeplitz structure of $\mathbf{H}_{f}$ allows us to write $\mathbf{H}_{f}=\sum_{k=0}^{L}\left(J^{k} \mathbf{S}\right) \otimes \mathbf{H}(k)$, and hence

$$
\begin{aligned}
\mathbf{R}_{f} & =\left(\sum_{k=0}^{L}\left(\mathbf{J}^{k} \mathbf{S}\right) \otimes \mathbf{H}(k)\right)\left(\sum_{l=0}^{L}\left(\mathbf{J}^{l} \mathbf{S}\right) \otimes \mathbf{H}(l)\right)^{*} \\
& =\sum_{k=0}^{L} \sum_{l=0}^{L}\left(\left(\mathbf{J}^{k} \mathbf{S}\right) \otimes \mathbf{H}(k)\right)\left(\left(\mathbf{S}^{T}\left(\mathbf{J}^{T}\right)^{l}\right) \otimes \mathbf{H}(l)^{*}\right) \\
& =\sum_{k=0}^{L} \sum_{l=0}^{L}\left(\mathbf{J}^{k} \mathbf{S S}^{T}\left(\mathbf{J}^{T}\right)^{l}\right) \otimes\left(\mathbf{H}(k) \mathbf{H}(l)^{*}\right) .
\end{aligned}
$$

The following proposition, whose proof is given in Appendix 1, shows that the matrix $\mathbf{J}^{k} \mathbf{S} \mathbf{S}^{T}\left(\mathbf{J}^{T}\right)^{l}$ has special structures that allows for the decomposition of (3.2) into a group of decoupled equations.

Proposition 3.1: Let $0 \leq k, l \leq L$ be two non-negative integers. For $l=k+j$, where $0 \leq j \leq L-k$, the upper triangular part of $\mathbf{J}^{k} \mathbf{S} \mathbf{S}^{T}\left(\mathbf{J}^{T}\right)^{l}$ is zero with only the $j$ th upper diagonal nonzero and is given by

$$
\Gamma_{j}\left(\mathbf{J}^{k} \mathbf{S S}^{T}\left(\mathbf{J}^{T}\right)^{l}\right)=\mathbf{q}_{k}(1: N-j, 1) \in \mathbb{R}^{N-j},
$$

where $\mathbf{q}_{k}=\mathbf{J}^{k} \mathbf{q}_{0}, 0 \leq k \leq L-j$, and $\mathbf{q}_{0}=$ $[\underbrace{11 \ldots 1} \underbrace{00 \ldots 0}]^{T} \in \mathbb{R}^{N}$.

$(N-L)$ entries Lentries

Since $\Upsilon_{j}\left(\left(\mathbf{J}^{k} \mathbf{S S}^{T}\left(\mathbf{J}^{T}\right)^{l}\right) \otimes\left(\mathbf{H}(k) \mathbf{H}(l)^{*}\right)\right)=\Gamma_{j}\left(\mathbf{J}^{k} \mathbf{S S}^{T}\left(\mathbf{J}^{T}\right)^{l}\right)$ $\otimes \mathbf{H}(k) \mathbf{H}(l)^{*}$, it follows from (3.2) and (3.3) that for $0 \leq j \leq L, \Upsilon_{j}\left(\mathbf{R}_{f}\right)$ can be described as follows:

$$
\begin{aligned}
\Upsilon_{j}\left(\mathbf{R}_{f}\right) & =\Upsilon_{j}\left(\mathbf{H}_{f} \mathbf{H}_{f}^{*}\right) \\
& =\sum_{k=0}^{L} \sum_{l=0}^{L} \Upsilon_{j}\left(\left(\mathbf{J}^{k} \mathbf{S} \mathbf{S}^{T}\left(\mathbf{J}^{T}\right)^{l}\right) \otimes\left(\mathbf{H}(k) \mathbf{H}(l)^{*}\right)\right) \\
& =\sum_{k=0}^{L} \sum_{l=0}^{L} \Gamma_{j}\left(\mathbf{J}^{k} \mathbf{S} \mathbf{S}^{T}\left(\mathbf{J}^{T}\right)^{l}\right) \otimes \mathbf{H}(k) \mathbf{H}(l)^{*} \\
& =\sum_{k=0}^{L-j} \mathbf{q}_{k}(1: N-j, 1) \otimes \mathbf{H}(k) \mathbf{H}(k+j)^{*} \\
& =\sum_{k=0}^{L-j}\left(\mathbf{q}_{k}(1: N-j, 1) \otimes \mathbf{I}_{J}\right) \mathbf{H}(k) \mathbf{H}(k+j)^{*} \\
& =\mathbf{M}_{j} \mathbf{F}_{j},
\end{aligned}
$$

where $\mathbf{F}_{j}=\left[\left(\mathbf{H}(0) \mathbf{H}(j)^{*}\right)^{T}\left(\mathbf{H}(1) \mathbf{H}(j+1)^{*}\right)^{T} \ldots(\mathbf{H}(L-j)\right.$ $\left.\left.\mathbf{H}(L)^{*}\right)^{T}\right]^{T} \in \mathbb{C}^{J(L-j+1) \times J}$ is formed from the products of channel matrix taps of the form $\mathbf{H}(k) \mathbf{H}(k+j)^{*}$, and $\mathbf{M}_{j}=\left[\mathbf{q}_{0}(1: N-j, 1) \mathbf{q}_{1}(1: N-j, 1) \ldots \mathbf{q}_{L-j}(1:\right.$ $N-j, 1)] \otimes \mathbf{I}_{J} \in \mathbb{R}^{J(N-j) \times J(L-j+1)}$.

Since $N>L+1$, the $(L+1)$ equations in (3.4) are overdetermined and consistent. Since it can be checked that $\mathbf{M}_{j}$ is full column rank for $j=0,1, \ldots, L$ (see Appendix 2), the solution $\mathbf{F}_{j}$ can be obtained as

$$
\mathbf{F}_{j}=\left(\mathbf{M}_{j}^{T} \mathbf{M}_{j}\right)^{-1} \mathbf{M}_{j}^{T} \Upsilon_{j}\left(\mathbf{R}_{f}\right), \quad j=0,1, \ldots, L .
$$

Let $\mathbf{Q}$ be the Hermitian matrix defined by $\mathbf{Q}=\mathbf{H H}^{*}$. Then we obtain $\mathbf{Q}$ from (3.5) since $\mathbf{Q}$ is Hermitian and $\Upsilon_{j}(\mathbf{Q})=\mathbf{F}_{j}$ for $j=0,1, \ldots, L$. Since $\operatorname{rank}(\mathbf{H})=K$ by assumption (B), $\mathbf{Q}$ has rank $K$. Since $\mathbf{Q}$ is Hermitian and positive semidefinite, $\mathbf{Q}$ has $K$ positive eigenvalues, say, $\lambda_{1}, \ldots, \lambda_{K}$. We can expand $\mathbf{Q}$ as

$$
\mathbf{Q}=\sum_{j=1}^{K}\left(\sqrt{\lambda_{j}} \mathbf{d}_{j}\right)\left(\sqrt{\lambda_{j}} \mathbf{d}_{j}\right)^{*},
$$

where $\mathbf{d}_{j}$ is a unit norm eigenvector of $\mathbf{Q}$ associated with $\lambda_{j}>0$. We can thus choose the channel impulse response matrix to be

$$
\widehat{\mathbf{H}}=\left[\begin{array}{lll}
\sqrt{\lambda_{1}} \mathbf{d}_{1} \sqrt{\lambda_{2}} \mathbf{d}_{2} & \ldots \sqrt{\lambda_{K}} \mathbf{d}_{K}
\end{array}\right] \in \mathbb{C}^{J(L+1) \times K} .
$$

We note $\mathbf{H}$ can only be identified up to a unitary matrix ambiguity $\mathbf{U} \in \mathbb{C}^{K \times K}[8]$, i.e., $\widehat{\mathbf{H}}=\mathbf{H U}$, since $\widehat{\mathbf{H}} \widehat{\mathbf{H}}^{*}=\mathbf{H H}^{*}=\mathbf{Q}$. The ambiguity matrix can be solved using a short training sequence [21]. 


\subsection{Discussions}

(1) The noisy case: When noise is present, the received covariance matrix becomes $\mathbf{R}_{f}=\mathbf{H}_{f} \mathbf{H}_{f}^{*}+\sigma_{w}^{2} \mathbf{I}_{J N}$ based on (2.1). Since the matrix $\mathbf{H}_{f} \in \mathbb{C}^{J N \times K M}$ is of full column rank, the rank of $\mathbf{H}_{f} \mathbf{H}_{f}^{*}$ is $K M$. This implies that the assoicated smallest $\left(J N-K M\right.$ ) eigenvalues of $\mathbf{R}_{f}$ are equal to the noise variances $\sigma_{w}^{2}$. Hence, in practice we can obtain the estimated noise variance $\hat{\sigma}_{w}^{2}$ as the average of the smallest $(J N-K M)$ eigenvalues of the sample covariance matrix $\widehat{\mathbf{R}}_{f}$. Then the proposed channel estimation algorithm can directly apply by substracting $\hat{\sigma}_{w}^{2} \mathbf{I}_{J N}$ from $\widehat{\mathbf{R}}_{f}$. Alternatively, $\sigma_{w}^{2} \mathbf{I}_{J N}$ can also be estimated via the method given in [7].

(2) Channel identifiability: For the proposed method, the channel identifiability condition is assumption (B), i.e., $\operatorname{rank}(\mathbf{H})=K$. Hence the channel needs not be irreducible, column reduced [8], or full column rank of $\mathbf{H}(0)$ required in the subspace method ([21], p. 1422). Thus the channel identifiability of the proposed method is more relaxed than that of the subspace method.

(3) Application to the more-input case: A necessary condition for the concatenated channel matrix $\mathbf{H}$ to be of full column rank (assumption $(\mathbf{B})$ ) is

$$
J(L+1) \geq K
$$

i.e., the product of the number of receive antennae $(J)$ and the channel length $(L+1)$ should be no less than the number of transmit antennae $(K)$. Hence, unlike the subspace method [21], which is exclusive for the more-output case, the proposed method is also capable of identifying more-input channels $(K>J)$, as long as the condition (3.8) is fulfilled.

(4) Computational complexity: Compared with the subspace method [21], the proposed method requires fewer computations. Detailed flop counts for these two methods are given in Appendix 3.

(5) Algorithm: We now summarize the proposed approach as the following algorithm:

(1) Collect the received data as $\overline{\mathbf{x}}(i)$, and then estimate the covariance matrix $\mathbf{R}_{f}$ via the following time average

$$
\widehat{\mathbf{R}}_{f}=\frac{1}{S} \sum_{i=1}^{S} \overline{\mathbf{x}}(i) \overline{\mathbf{x}}(i)^{*}
$$

where $S$ is the number of symbol blocks.

(2) Use the method given in this subsection to eliminate the noise component $\hat{\sigma}_{w}^{2} \mathbf{I}_{J N}$ imposing on $\widehat{\mathbf{R}}_{f}$ to get $\mathbf{R}_{c}=\widehat{\mathbf{R}}_{f}-\hat{\sigma}_{w}^{2} \mathbf{I}_{J N}$.
(3) Form the matrix $\mathbf{M}_{j}$ and compute $\mathbf{F}_{j}$ using (3.5) for $j=0,1, \ldots, L$. (Here we use $\mathbf{R}_{c}$ instead of $\mathbf{R}_{f}$ in (3.5).)

(4) Form the matrix $\mathbf{Q}$ using $\mathbf{F}_{0}, \mathbf{F}_{1}, \ldots, \mathbf{F}_{L}$, and obtain the channel impulse response matrix (3.7) by computing the $K$ largest eigenvalues and the associated eigenvectors of $\mathbf{Q}$.

\section{Simulation}

In this section, we use several numerical simulations to demonstrate the performance of the proposed method. We first consider two 2-input 2-output test channels, both with $L=2$. Channel A is shown as follows:

$$
\begin{aligned}
& \mathbf{H}(0)=\left[\begin{array}{ll}
0.36+0.21 j & 0.48+0.29 j \\
0.26+0.16 j & 0.5+0.17 j
\end{array}\right], \\
& \mathbf{H}(1)=\left[\begin{array}{cc}
-0.49-0.36 j & 0.93+0.06 j \\
0.88+1.30 j & 0.87+0.68 j
\end{array}\right], \\
& \mathbf{H}(2)=\left[\begin{array}{ll}
0.73-0.14 j & -0.13-0.27 j \\
0.29-0.4 j & -0.44-0.55 j
\end{array}\right],
\end{aligned}
$$

and channel B is the same as channel A except $\mathbf{H}(0)=$ $\left[\begin{array}{l}0.48+0.24 j 0.32+0.12 j \\ 0.24+0.13 j 0.18+0.06 j\end{array}\right]$. The length of symbol blocks is $M=18$, which is zero padded to blocks of length $M+$ $P=20$. It means $P=2(=L)$ and transmission efficiency is $90 \%$. The number of symbol blocks is $S=100$. The channel normalized mean-square error (NMSE) is defined as NMSE $=(1 / I) \sum_{i=1}^{I}\left\|\widehat{\mathbf{H}}^{(i)}-\mathbf{H}\right\|_{F}^{2} \cdot\|\mathbf{H}\|_{F}^{-2}$, where $I=$ 200 is the number of Monte Carlo runs, and $\|\cdot\|_{F}$ denotes the Frobenius norm. $\widehat{\mathbf{H}}^{(i)}=\left[\widehat{\mathbf{H}}^{(i)}(0)^{T} \widehat{\mathbf{H}}^{(i)}(1)^{T} \widehat{\mathbf{H}}^{(i)}(2)^{T}\right]^{T}$ is the $i$ th estimate of the channel impulse response matrix $\mathbf{H}$ after removing the unitary matrix ambiguity by the least squares method [8]. The input source symbols are i.i.d. QPSK signals. The SNR at the output is defined as $\mathrm{SNR}=\frac{E\left[\|\mathbf{x}(n)-\mathbf{w}(n)\|_{2}^{2}\right]}{E\left[\|\mathbf{w}(n)\|_{2}^{2}\right]}$. The channel noise is zero mean, temporally and spatially white Gaussian.

In the first experiment, we compare the performance of the proposed method with that of the subspace method [21]. Figures 2 and 3 are the simulation results of zeroforcing equalization for channels $\mathrm{A}$ and $\mathrm{B}$, respectively. Figure 2 shows that the proposed method outperforms the subspace method from low to medium SNR, whereas the subspace method gives better performance for high SNR; and Figure 3 shows that the performance of the proposed method is better than that of the subspace method. From Figures 2 and 3, we observe that the performance of the subspace method deteriorates for channel B. This is expected since by computation, the condition number of $\mathbf{H}(0)$ for channel $\mathrm{B}(=47.18)$ is larger than that of 


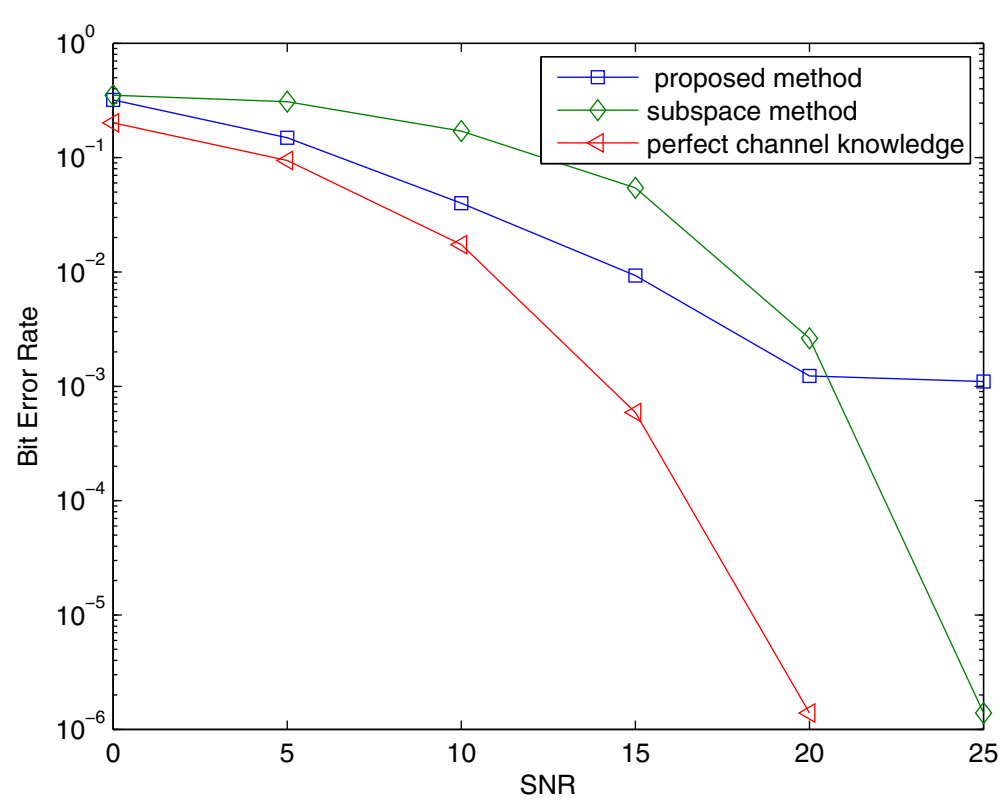

Figure 2 Channel A: Bit error rate versus output SNR.

channel $\mathrm{A}(=12.69)$, which means $\mathbf{H}(0)$ for channel $\mathrm{B}$ is closer to singularity (rank deficiency) and tends to violate the identifiability condition of the subspace method ([21], p. 1422).

In the second experiment, we generate 100 3-input 2output random channels with $L=2$ to illustrate the estimation performance of the proposed method for channels with more transmitters than receivers. We use $M=18$ and $P=2$. Each channel coefficient in the channel matrix is generated according to the independent complexedvalued Gaussian distribution with zero mean and unit variance. Figure 4 shows that for different numbers of symbol blocks, the proposed method is capable of identifying the more-input channels. In addition, the NMSE decreases as SNR increases and is roughtly constant for high SNR. A possible explanation is that for sufficiently

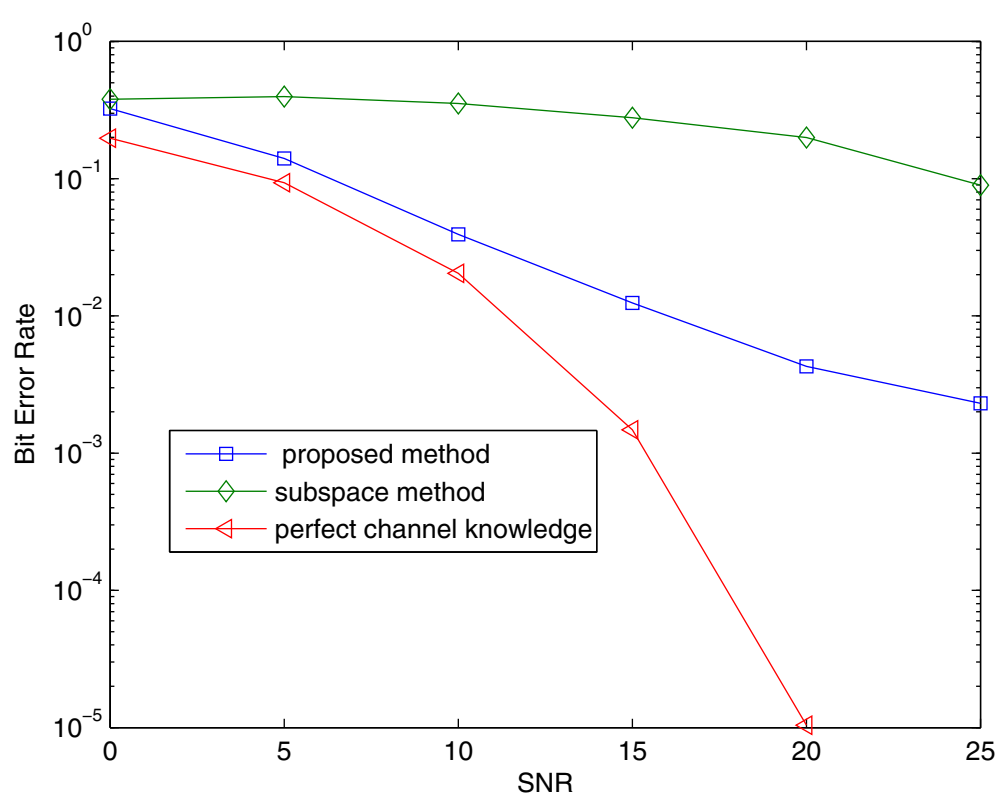

Figure 3 Channel B: Bit error rate versus output SNR. 


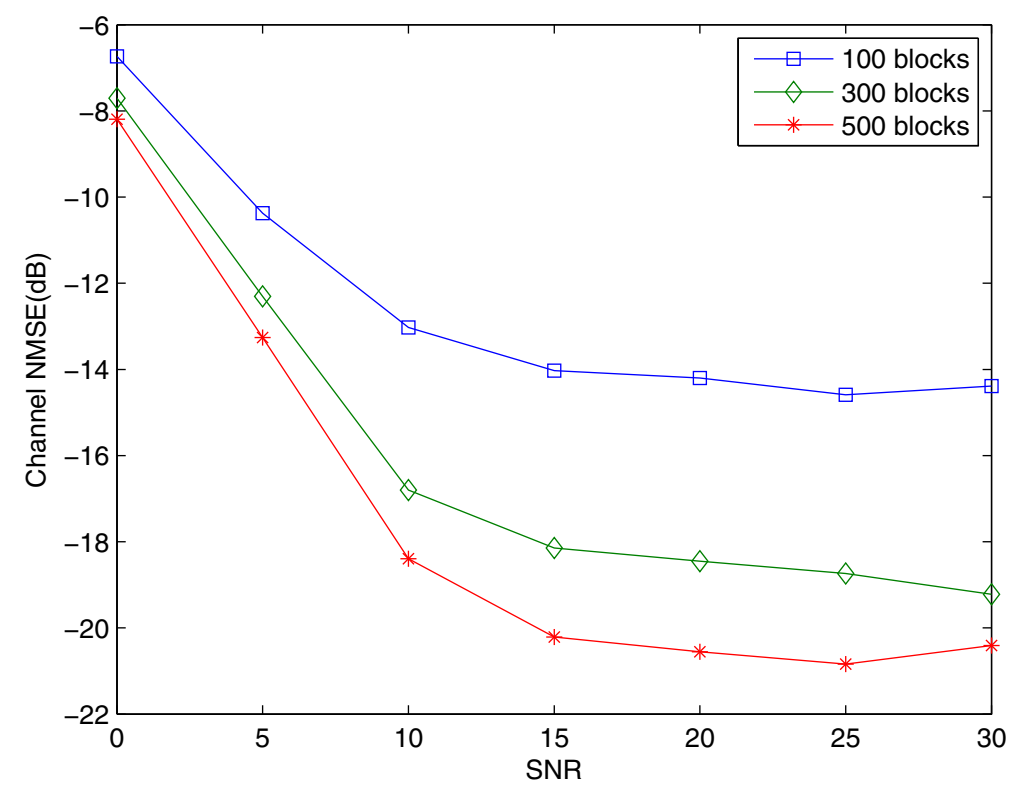

Figure 4 Channel NMSE versus SNR (more-input channel case).

high SNR, the channel NMSE is contributed mainly due to numerical error than by channel noise. The existence of the error floor at high SNR due to numerical error is a well-known result, and this common phenomenon can also be observed in some previous works related to blind channel estimation [9-14].

In the third experiment, we apply the proposed method to 2002 -input 4-out random channels with $L=4$ to demonstrate the performance. We use $M=36$ and $P=$ 4 to maintain the transmission efficiency at $90 \%$. Each channel coefficient in the channel matrix is still generated according to the independent complexed-valued Gaussian distribution with zero mean and unit variance. Figures 5 and 6 show that as the number of symbol blocks (used to obtain the covariance matrix $\widehat{\mathbf{R}}_{f}$ ) increases from 200 to 400 , the BER approaches to the ideal case. In addition,

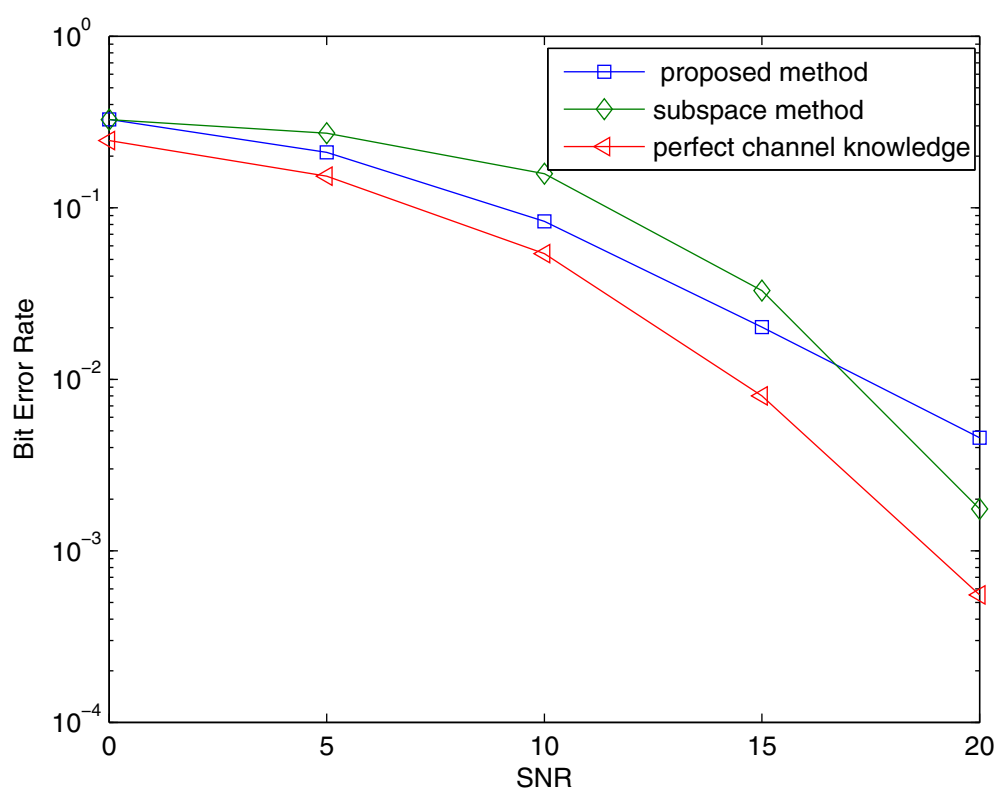

Figure 5 Bit error rate versus output SNR (200 symbol blocks). 


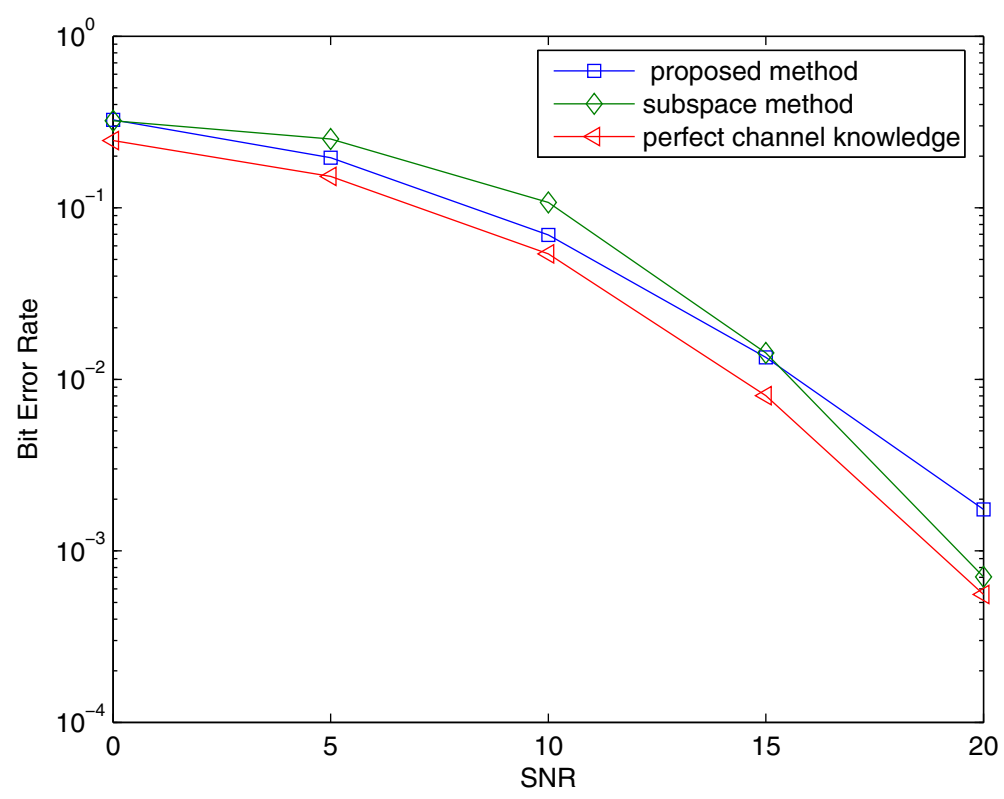

Figure 6 Bit error rate versus output SNR (400 symbol blocks).

these two figures also show that the proposed method outperforms the subspace method from low to medium SNR, and the subspace method performs better for high SNR.

In the literature of blind channel estimation, it is wellknown that subspace methods, such as $[18,21]$, enjoy the so-called "finite sample convergence" property [15-19,21], that is, in the noiseless case (or sufficicently high SNR), the channels can be almost exactly identified by using a finite number of samples for covariance estimation. This is the reason why the subspace-based solution can yield improved channel estimation accuracy and the resultant BER in the high SNR region. The proposed method, like most of other solution branches, e.g., [9-14], can be classified as the "SCM" approach, by which exact channel identification is achieved whenever the exact covariance matrix is available. Hence, it is not unexpected that our method is susceptible to finite-sample errors in covariance matrix estimation, which leads to an MSE floor in the high SNR region. Such a phenomenon is not uncommon in the literature, and has been seen in many studies, e.g., [9-14]. Despite this, the proposed method can outperform the subspace algorithm in the low SNR region, and hence could be a potential candidate in harsh communication environments.

\section{Conclusion}

We propose an SCM based blind channel estimation method for zero padding MIMO-OFDM systems. By exploiting the block Toeplitz channel matrix structure, we solve the channel product matrices from a series of decoupled linear equations obtained from the covariance matrix of the received data. Then the channel impulse response matrix can be obtained by taking eigen-decomposition of a Hermitian matrix formed from the channel product matrices. The identifiability condition is more relaxed than that of the subspace method [21]. Unlike most of existing solutions that are only applicable for the more-output channels, the proposed approach can also identify the more-input channels under a quite mild condition. Simulation results are used to demonstrate the performance of the proposed method. Compared with the subspace method [21], the proposed method is shown to have better performance form low to medium SNR or when $\mathbf{H}(0)$ tends to be singular.

\section{Appendix 1: Proof of Proposition 3.1}

Let $\mathbf{e}_{n}$ be the $n$th column of the identity matrix $\mathbf{I}_{N}$. Then for $k=0$ case,

$$
\begin{aligned}
& \Gamma_{j}\left(\mathbf{J}^{0} \mathbf{S} \mathbf{S}^{2}\left(\mathbf{J}^{T}\right)^{j}\right) \\
& =\Gamma_{j}\left(\left[\mathbf{e}_{1} \mathbf{e}_{2} \ldots \mathbf{e}_{N-L} \mathbf{0} \ldots \mathbf{0}\right]\left(\mathbf{J}^{T}\right)^{j}\right) \\
& =\left\{\begin{array}{l}
\Gamma_{0}\left(\left[\mathbf{e}_{1} \mathbf{e}_{2} \ldots \mathbf{e}_{N-L} \mathbf{0} \ldots \mathbf{0}\right]\right)=\mathbf{q}_{0} \\
\Gamma_{j}\left([\underbrace{\mathbf{0} \ldots \mathbf{0} \mathbf{e}_{1} \mathbf{e}_{2} \ldots \mathbf{e}_{N-j} \mathbf{e}_{N-j+1} \ldots \mathbf{e}_{N-L} \mathbf{0} \ldots \mathbf{0}}_{j \text { columns }}]\right) \\
=\mathbf{q}_{0}(1: N-j, 1), 1 \leq j \leq L .
\end{array}\right.
\end{aligned}
$$

Hence $\Gamma_{j}\left(\mathbf{J}^{0} \mathbf{G}^{2}\left(\mathbf{J}^{T}\right)^{j}\right)=\mathbf{q}_{0}(1: N-j, 1)$ for $0 \leq j \leq L$. 
For $k=1$ case,

$$
\begin{aligned}
& \Gamma_{j}\left(\mathbf{J}^{1} \mathbf{S S}^{T}\left(\mathbf{J}^{T}\right)^{1+j}\right) \\
& =\Gamma_{j}\left(\mathbf{J}\left[\mathbf{e}_{1} \mathbf{e}_{2} \ldots \mathbf{e}_{N-L} \mathbf{0} \ldots \mathbf{0}\right]\left(\mathbf{J}^{T}\right)^{1+j}\right) \\
& =\Gamma_{j}\left(\left[\mathbf{e}_{2} \mathbf{e}_{3} \ldots \mathbf{e}_{N-L+1} \mathbf{0} \ldots \mathbf{0}\right]\left(\mathbf{J}^{T}\right)^{1+j}\right)
\end{aligned}
$$

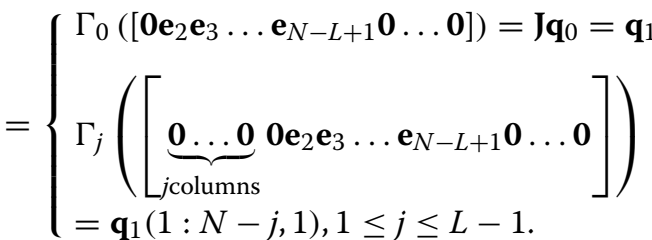

Hence $\Gamma_{j}\left(\mathbf{J}^{1} \mathbf{G}^{2}\left(\mathbf{J}^{T}\right)^{1+j}\right)=\mathbf{q}_{1}(1: N-j, 1)$ for $0 \leq j \leq L$.

Following the same process, we can obtain the cases $\Gamma_{j}\left(\mathbf{J}^{k} \mathbf{G}^{2}\left(\mathbf{J}^{T}\right)^{k+j}\right)=\mathbf{q}_{k}(1: N-j, 1)$ for $k=2,3, \ldots, L$, through some straightforward manipulation, and assert the result given in Proposition 3.1.

\section{Appendix 2: Proof of full column rank of $\mathrm{M}_{\boldsymbol{j}}$}

Let $\mathbf{P}_{j}=\left[\mathbf{q}_{0}(1: N-j, 1) \mathbf{q}_{1}(1: N-j, 1) \ldots \mathbf{q}_{L-j}(1:\right.$ $N-j, 1)] \in \mathbb{R}^{(N-j) \times(L-j+1)}$ for $j=0,1, \ldots, L$. We note that $\mathbf{P}_{0}$ is a Toeplitz matrix with $\mathbf{q}_{0}$ being its first column and $[100 \ldots 0] \in \mathbb{R}^{1 \times(L+1)}$ being its first row. Hence $\mathbf{P}_{0}$ is full column rank.

Also we observe that for $j=1,2, \ldots, L, \mathbf{P}_{j}$ is obtained from $\mathbf{P}_{0}$ by deleting its last $j$ rows and the last $j$ columns, i.e., $\mathbf{P}_{j}$ is a Toeplitz matrix with $\mathbf{q}_{0}(1: N-j, 1)$ being its first column and $[1 \underbrace{00 \ldots 0}_{L-j}] \in \mathbb{R}^{1 \times(L-j+1)}$ being its first row. Thus, for $j=1,2, \ldots, L, \mathbf{P}_{j}$ is full column rank.

Since $\mathbf{P}_{j}$ is full column rank, $\mathbf{M}_{j}=\mathbf{P}_{j} \otimes \mathbf{I}_{j}$ is full column rank for $j=0,1, \ldots, L$.

\section{Appendix 3: Complexity evaluation}

The proposed method is compared with the subspace method [21] in terms of flops, where a "flop" is defined to be a single complex multiplication or addition [22].

Proposed method: Estimate the (Hermitian) covariance matrix $\mathbf{R}_{f}$ using (3.9); this requires $(2 S-1) \frac{J N(J N+1)}{2}+1$ flops. Estimate and eliminate the noise variance to obtain $\mathbf{R}_{c}$ needs $3 J N$ flops. Solving $(L+1)$ least square problems using QR factorization ([22], p. 254) requires $2 J^{3} \sum_{j=0}^{L}(L-$ $j+1)^{2}\left[N-j-\frac{L-j+1}{3}\right]$ flops. Eigen-decomposition of a $J(L+1) \times J(L+1)$ requires $12 J^{3}(L+1)^{3}$ flops.

Subspace method: Estimate the covariance matrix requires $(2 S-1) \frac{J N(J N+1)}{2}+1$ flops. Eigen-decomposition of a $J(L+M) \times J(L+M)$ matrix requires $12 J^{3}(L+M)^{3}$ flops. Singular value decomposition of a $(J N-K M) N \times J(L+1)$ matrix $([22]$, p. 240) requires $4 J^{2}(L+1)^{2}\left[J\left(2 L+2+N^{2}\right)-K M N\right]$ flops.
According to the above flop computation, for the first experiment simulation in Section 4, the proposed method and the subspace method require about $1.7 \times 10^{5}$ flops and $9.44 \times 10^{5}$ flops, respectively. For experiment 3 using 200 symbol blocks, the proposed method and the subspace method require about $5.5 \times 10^{6}$ flops and $5.99 \times 10^{7}$ flops, respectively.

Competing interests

The authors declare that they have no competing interests.

\section{Acknowledgements}

The research was sponsored by the National Science Council of Taiwan under grant NSC 99-2221-E-035-056-

\section{Author details}

${ }^{1}$ Feng Chia University, Taichung, Taiwan. ${ }^{2}$ National Chiao Tung University, Hsinchu, Taiwan.

Received: 29 October 2011 Accepted: 6 June 2012

Published: 12 July 2012

\section{References}

1. Z Wang, GB Giannakis, Wireless multicarrier communications: where Fourier meets Shannon. IEEE Signal Process. Mag. 17(3), 29-48 (2000)

2. GL Stüber, JR Barry, SW Mclaughlin, Y Li, MA Ingram, TG Pratt, Broadband MIMO-OFDM wireless communications. Proc. IEEE. 92(2), 271-294 (2004)

3. GB Giannakis, Y Hua, P Stoica, L Tong, Signal Processing, Advances in Wireless and Mobile Communications Volume l: Trends in Channel Identification and Equalization (Prentice Hall, PTR, Upper Saddle River, 2001)

4. YS Chen, CA Lin, Blind-channel identification for MIMO single-carrier zero-padding block-transmission systems. IEEE Trans. Circ. Syst.-I: Regular Papers. 55(6), 1571-1579 (2008)

5. X Zhuang, Z Ding, AL Swindlehurst, A statistical subspace method for blind channel identification in OFDM communications. in Proc. IEEE Int Conf. Acoust. Speech Signal Process, 5, (Philadelphia, Pennsylvania, USA, 2005), pp. 2493-2496

6. FD Backx, TTV Vinhozaand, R Sampaio-Neto, Blind channel estimation for zero-padded OFDM systems based on correlation matching. in Proc. IEEE Vehicular Technology Conference-Fall, (Baltimore, Maryland, USA, 2007), pp. 1308-1311

7. YS Chen, CA Lin, Blind identification of MIMO channels in zero padding block transmission systems. IEEE Trans. Signal Process. 55(2), 764-772 (2007)

8. Z Ding, Matrix outer-product decomposition method for blind multiple channel identification. IEEE Trans. Signal Process. 45(12), 3053-3061 (1997)

9. C Shin, RW Heath Jr., EJ Powers, Non-redundant precoding-based blind and semi-blind channel estimation for MIMO block transmission with a cyclic prefix. IEEE Trans. Signal Process. 56(6), 2509-2523 (2008)

10. Z Ding, L Qiu, Blind MIMO channel identification from second order statistics using rank deficient channel convolution matrix. IEEE Trans. Signal Process. 51(2), 535-544 (2003)

11. F Gao, A Nallanathan, Blind channel estimation for MIMO OFDM systems via nonredundant linear precoding. IEEE Trans. Signal Process. 55(2), 784-789 (2007)

12. F Gao, A Nallanathan, Blind channel estimation for OFDM systems via a generalized precoding. IEEE Trans. Veh. Technol. 56(3), 1155-1164 (2007)

13. FJ Simois, JJ Murillo-Fuentes, R Boloix-Tortosa, L Salamanca, Near the Cramér-Rao bound precoding algorithms for OFDM blind channel estimation. IEEE Trans. Veh. Technol. 61(2), 651-661 (2012)

14. V Khanagha, A Khanagha, VT Vakili, Modified particle swarm optimization for blind deconvolution and identification of multichannel FIR filters. EURASIP J. Avd. Signal Process. 2010, 716862 (2010)

15. L Tong, Q Zhao, Joint order detection and blind channel estimation by least squares smoothing. IEEE Trans. Signal Process. 47(9), 2345-2355 (1999)

16. Q Zhao, L Tong, Adaptive blind channel estimation by least squares smoothing. IEEE Trans. Signal Process. 47(12), 3000-3012 (1999)

17. $X Y u, L$ Tong, Joint channel and symbol estimation by oblique projections. IEEE Trans. Signal Process. 49(12), 3074-3083 (2001) 
18. E Moulines, P Duhamel, JF Cardoso, S Mayrargue, Subspace methods for the blind identification of multichannel FIR filters. IEEE Trans. Signal Process. 43(2), 516-525 (1995)

19. GXu, H Liu, L Tong, T Kailath, A least-squares approach to blind channel identification. IEEE Trans. Signal Process. 43(12), 2982-2993 (1995)

20. A Scaglione, GB Giannakis, S Barbarossa, Redundant filter bank precoders and equalizers-Part II: blind channel estimation, synchronization, and direct equalization. IEEE Trans. Signal Process. 47(7), 2007-2022 (1999)

21. Y Zeng, T-Y Ng, A semi-blind channel estimation method for multiuser multiantenna OFDM systems. IEEE Trans. Signal Process. 52(5), 1419-1429 (2004)

22. GH Golub, CF Van Loan, Matrix Computions, 3rd edn. (The Johns Hopkins University Press, Baltimore, 1996)

\section{Submit your manuscript to a SpringerOpen ${ }^{\circ}$ journal and benefit from:}

- Convenient online submission

- Rigorous peer review

- Immediate publication on acceptance

- Open access: articles freely available online

- High visibility within the field

- Retaining the copyright to your article 